\title{
BRILLOUIN ZONE MAPPING OF THE EXISTENCE CONDITIONS FOR INTERFACE BILAYER SPIN-WAVES.
}

EFFECTS OF THE CONFIGURATION OF THE FILM MAGNETIZATION

\author{
H. Puszkarski $I^{a, b}$, R. Józefowicz ${ }^{a}$, B. KolodziejCzAK ${ }^{a}$, A. AKJouj ${ }^{b}$, \\ B. Djafari-Rouhani ${ }^{b}$ and L. Dobrzynski ${ }^{b}$ \\ ${ }^{a}$ Surface Physics Division, Physics Institute, A. Mickiewicz University \\ Matejki 48/49, 60-769 Poznań, Poland \\ b Laboratoire de Dynamique et Structure des Matériaux Moléculaires \\ Unité Associée au CNRS 801, Université des Sciences et Technologies de Lille I \\ 59655 Villeneuve d'Ascq, Cedex, France.
}

(Received January 12, 1996)

For the two interface cubic cuts: $\operatorname{sc}(110)$ and sc(111) we consider the existence regions of interface spin-waves of a ferromagnetic bilayer film on the two-dimensional Brillouin zone as to their size and shape versus the respective interface related parameters (interface exchange coupling and intrinsic interface anisotropy) applying the approximation of a very thick bilayer film and the method of Brillouin zone mapping (the three (100) interface cubic orientations have been dealt with earlier [Phys. Rev. B 51, 16008 (1995)]). Of particular interest to us still remains the emergence of interface spin-waves induced by varying dynamic quantities such as the in-plane propagation wave vector $\boldsymbol{k}_{\|}$. Moreover, in our present investigation, we put especial emphasis on the effect exerted on the Brillouin zone mapping by varying (in the plane perpendicular to the film) the configuration angle $\vartheta$ of the film magnetization with respect to the film normal. We predict the existence of (at the most two) critical angles $\vartheta_{c}$ at which the interface spin-waves emerge. These critical angles are functions of the in-plane wave vector $k_{\|}$of interface spin-wave propagation along the interface. Finally, we discuss the effects due to the presence of critical angles with a view to their exploitation in experimental measurements.

PACS numbers: 75.30.Ds, 75.70.Cn

\section{Introduction}

In our preceding paper [1] (to be referred to henceforth as Part I) we applied a Brillouin zone mapping (BZM) [2] visualizing the regions of existence of interface spin-waves (ISWs) on the two-dimensional Brillouin zone (2D BZ); we considered 
the three interface cubic orientations $\operatorname{sc}(100), f c c(100)$ and $b c c(100)$ witipin the framework of the model of a ferromagnetic bilayer film described by a standard Heisenberg Hamiltonian, admitting arbitrary (with respect to the film normal) configurations of the film magnetization, arbitrary (ferro- or antiferromagnetic) interface exchange coupling and arbitrary (easy-axis/easy-plane) uniaxial interface anisotropy. Exact conditions for the existence of ISWs were established in the limit of very thick bilayer film; we put emphasis on this particular case since it enabled us to find analytically the exact expression for the ISW energy and then to study it versus the respective interface parameters.

In this paper we extend our work to a detailed analysis of the other two interface cubic cuts, namely: sc(110) and sc(111); we proceed within the framework of the same model for the bilayer ferromagnetic film as considered in Part I (also the BZM will be presented in the same limit of a very thick bilayer film, as before). Although our present work was initially aimed at completing our earlier analysis, it turned out to lead far beyond the results of Part I: we analyze the conditions for ISW existence in their dependence on the configuration angle $\vartheta$ between the film magnetization and the film normal. We show how the BZM regions of existence of ISWs on the BZ vary with the film magnetization orientation $\vartheta$, and we predict the existence of a critical angle $\vartheta_{c}$ on traversal of which an ISW emerges. We believe this effect, consisting in the configurational induction of ISW's, may prove a highly potent tool for experimenters since the control of the film magnetization angle $\vartheta$ is one of the easiest manipulations performable on magnetic samples.

\section{Model}

In this section we re-state the essentials of our model presented in Part I. We simultaneously adapt the respective formulas to the cases to be considered in the present paper, but keep all notations of Part I unchanged.

We study a magnetic system in the form of a bilayer film composed of two ferromagnetic layers (sublayers $A$ and $B$ ) of the same magnetic material, coupled by exchange interactions at their interface. We write the Hamiltonian of our bilayer film as follows:

$$
\widehat{\mathcal{H}}=-\sum_{l j \neq l^{\prime} j^{\prime}} J_{l l^{\prime}} \widehat{S}_{l} j \cdot \widehat{S}_{l}^{\prime} j^{\prime}-g \mu_{\mathrm{B}} \sum_{l j} H^{\mathrm{eff}} \cdot \widehat{S}_{l j}-\sum_{l j} D_{l}\left(\widehat{S}_{l} j^{z}\right)^{2}
$$

with terms accounting successively for the isotropic exchange interactions, the Zeeman energy of the spins, and the uniaxial (single-ion) interface anisotropy energy. We write for the NN exchange terms $J_{l l^{\prime}}=J$ if both spins belong to the same sublayer and $J_{l l^{\prime}}=J^{A B}$ if the spins are coupled across the interface between the sublayers $A$ and $B$; the ratio of the two integrals is $J_{\text {int }} \equiv J^{A B} / J$. We assume that the externally applied static magnetic field $H$ is oriented at some angle to the film normal (assumed as the $z$-axis) and can be rotated in the plane perpendicular to the film surface. The bulk effective field $\boldsymbol{H}^{\text {eff }}$ acting on a given spin is defined as the sum of the external static field and the demagnetization as well as the bulk anisotropy fields and is assumed to be homogeneous throughout the film. The third (anisotropic) term of (1) comprises the interface anisotropy effect, specified as $D_{l}=0$ for surface and bulk spins, and $D_{l}=D^{\prime}$ for interface spins. We 
denote the wave vector component of the spin waves (excitations resulting from the Iamiltonian (1)) parallel to the surface by $k_{\|}=\left[k_{1}, k_{2}\right]$. The spin waves will additionally be characterized by a third wave vector component $k_{3} \equiv k$ in the $z$ direction normal to the film surface; one should note that our quantity $k$ is a reduced wave vector, i.e. its components are expressed in lattice units.

Throughout the present paper we focus our attention exclusively on acoustical interface-localized spin-waves, i.e. on those solutions for which $k$ is purely imaginary: $k=\mathrm{i} t$, with $t$ a real and positive number. The reduced energy of ISW (as defined in Part I, Eq. (10)) is expressed by the quantity $t$ as follows:

$$
E_{\text {red }}^{\mathrm{ISW}}=\left(z_{\|}+2 z_{\perp}\right)-\Gamma_{\|}^{k_{\|}}-2\left|\Gamma_{\perp}^{k_{\|}}\right| \cosh t .
$$

The function $\Gamma_{\|}^{k_{\| 1}}$ is a term modulating the energy spectrum but not affecting the structure of the spectrum so that its presence will be of no particular importance in our analysis. It is the function $\left|\Gamma_{\perp}^{k_{\|}}\right|$which exerts the decisive influence on the behaviour of our model since only this function appears simultaneously in the expressions for the energy as well as the interface parameters. In the limit of the very thick film (i.e. when the thickness of the film is made to grow infinitely) we found the following two exact roots:

$$
2 \cosh t=\left(b \pm J_{\text {int }}\right)+\left(b \pm J_{\text {int }}\right)^{-1}
$$

which correspond to two ISWs, which can possibly appear in the bilayer spin-wave spectrum. Above, we have used the following notations:

$$
b=\frac{z_{\perp}}{\left|\Gamma_{\perp}^{k_{\text {ill }}}\right|}\left[1-J_{\text {int }}-D_{\text {int }}\left(3 \cos ^{2} \vartheta-1\right)\right], \quad D_{\text {int }}=\frac{D^{\prime}(S-1 / 2)}{2 S J z_{\perp}}
$$

where $\vartheta$ is the angle between the direction of the film magnetization and that of the normal to the surface of the film, $z$ is the number of nearest neighbours situated in the same plane $\left(z_{\|}\right)$or in an adjacent plane $\left(z_{\perp}\right)$. We shall present here the results obtained for cubic crystals with the two interface film cuts not considered in Part I; the respective structural factors, appearing in Eqs. (2-4), take the following forms for either case [3-5]:

$$
z_{\perp}=2, \quad\left|\Gamma_{\perp}^{k_{\| 1}}\right|=2 \cos \frac{1}{2} k_{2} ; \quad z_{\|}=2, \quad \Gamma_{\|}^{k_{\| 1}}=2 \cos k_{1}
$$

for $\operatorname{sc}(110)$ interface cut,

$$
\begin{aligned}
& z_{\perp}=3, \quad\left|\Gamma_{\perp}^{k_{\| 1}}\right|=\left\{3+2\left[\cos k_{1}+\cos k_{2}+\cos \left(k_{1}+k_{2}\right)\right]\right\}^{1 / 2} ; \\
& z_{\|}=0, \quad \Gamma_{\|}^{k_{\|}}=0 \\
& \text { for sc(111) interface cut, }
\end{aligned}
$$

where $k_{1}, k_{2}$ are the in-plane wave-vector components in directions determined against the background of the respective two-dimensional Brillouin zone, as shown in Fig. 1.

The general condition for the existence of ISWs remains as in Part I and requires the following inequalities to be fulfilled:

$$
\frac{z_{\perp}}{\left|\Gamma_{\perp}^{k_{\text {il }}}\right|}\left[1-D_{\text {int }}\left(3 \cos ^{2} \vartheta-1\right)-J_{\text {int }}\right] \geq 1 \mp J_{\text {int }} .
$$



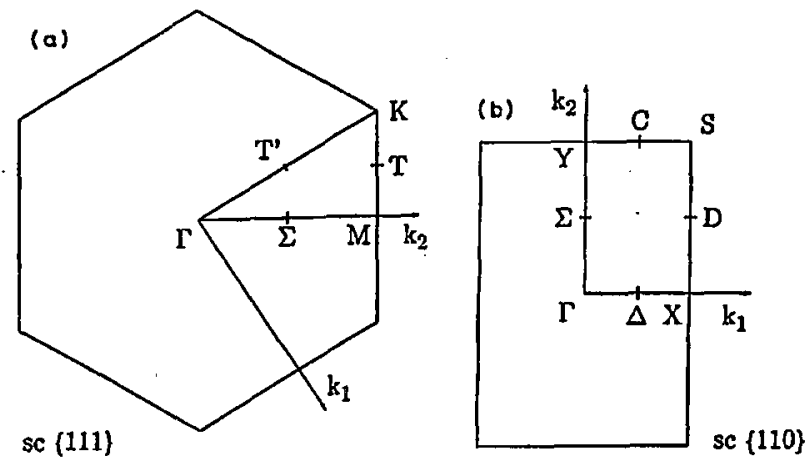

Fig. 1. Two-dimensional interface Brillouin zones for interface cuts in cubic structures: (a) $\operatorname{sc}(111)$ and (b) sc(110) interfaces.

One notes that these conditions are functions of the in-plane spin-wave propagation vector $k_{\|}$by way of the structural factor $\left|\Gamma_{\perp}^{k_{\|}}\right|$. In what follows we illustrate the dependence of the above existence conditions for ISWs on $k_{\|}$by determining the appropriate regions of ISW existence on the 2D BZ (BZM [2]); the essence of BZM is to determine graphically those directions of $k_{\|}$in-plane propagations for which ISWs exist. We start with the case of $\operatorname{sc}(111)$.

\section{Case of sc(111) interface cut}

Using Eqs. (2-4) and (5b) we get the following explicit expressions for the reduced ISW energy:

$$
E_{\text {red }}^{\mathrm{ISW}}[\operatorname{sc}(111)]=6-B_{ \pm}-\frac{3+2\left[\cos k_{1}+\cos k_{2}+\cos \left(k_{1}+k_{2}\right)\right]}{B_{ \pm}}
$$

where we have made use of the following notation:

$$
\begin{aligned}
B_{ \pm} & =3\left[1-J_{\text {int }}-D_{\text {int }}\left(3 \cos ^{2} v-1\right)\right] \\
& \pm J_{\text {int }} \sqrt{3+2\left[\cos k_{1}+\cos k_{2}+\cos \left(k_{1}+k_{2}\right)\right]} .
\end{aligned}
$$

Similarly, by Eqs. (6) and (5b), we get the following conditions for the existence of ISWs:

$$
\frac{3\left[1-D_{\text {int }}\left(3 \cos ^{2} \vartheta-1\right)-J_{\text {int }}\right]}{\sqrt{3+2\left[\cos k_{1}+\cos k_{2}+\cos \left(k_{1}+k_{2}\right)\right]}} \geq 1 \mp J_{\text {int }} .
$$

Obviously, the fulfillment of either one of the two conditions (9) corresponds to the existence of one ISW. Thus, as in Part I, we can have 0 or 1 or at most 2 branches of ISWs (note that the upper and lower signs in Eqs. (7-9) are mutually correlated).

The Brillouin zone mapping of the existence regions of ISWs determined from the conditions (9) is shown in Fig. 2. Two cases occur, according to whether interface exchange coupling is ferromagnetic (Fig. 2a) or antiferromagnetic (Fig. 2b). Furthermore, in either case, we consider two sub-cases, according to whether the 

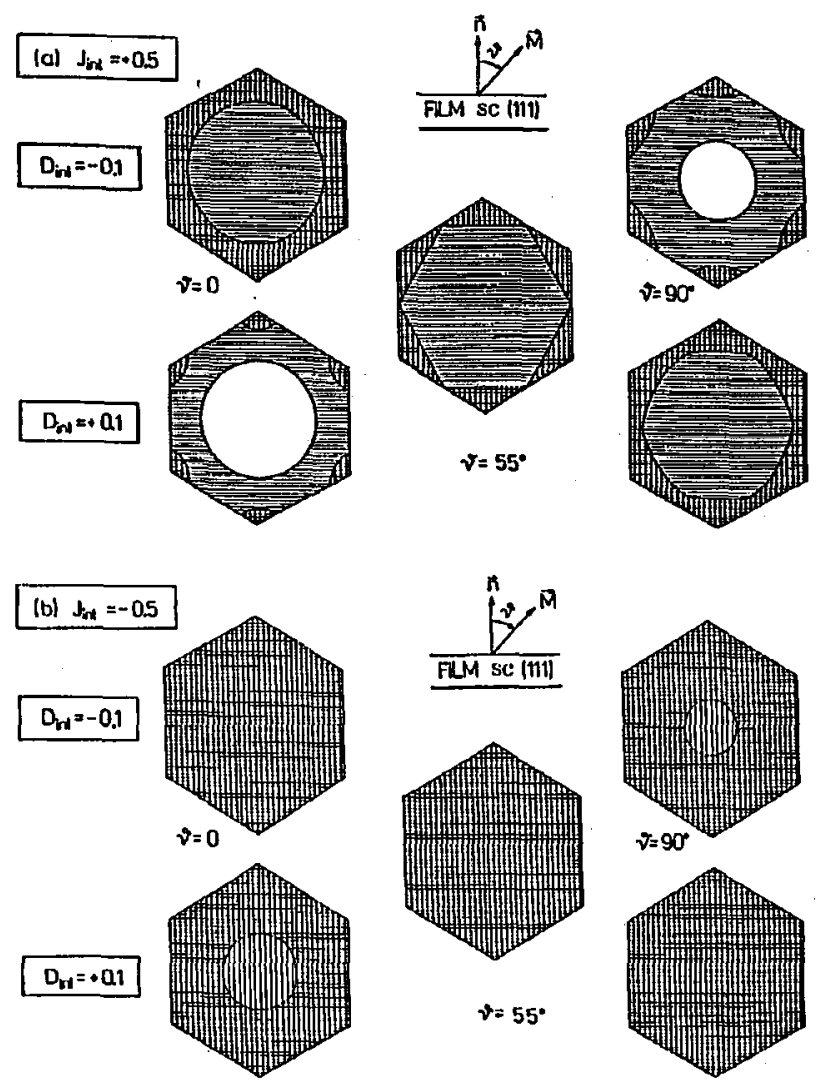

Fig. 2. Brillouin zone mapping of the regions of existence of acoustical bilayer interface spin-waves for sc(111) film with (a) ferromagnetic and (b) antiferromagnetic interface coupling. Two interface waves exist in the more densely shaded regions, one - in the less densely shaded regions, and none - in the unshaded areas. Notations: $J_{\text {int }}$-interface exchange coupling parameter, $D_{\text {int }}-$ uniaxial interface anisotropy constant, and $\vartheta$ the angle of film magnetization orientation. Note that for the case of $\vartheta \approx 55^{\circ}$ the mapping is insensitive to the intrinsic interface anisotropy $D_{\mathrm{int}}$.

interface anisotropy is of the easy-axis type, $D_{\text {int }}>0$, or respectively of the easy-plane type, $D_{\text {int }}<0$. For each of the four eventualities we investigate how the mapping of the regions of ISW existence varies with the orientation of the magnetization vector of the sample with respect to the film surface. In Fig. 2, the unshaded region corresponds to that portion of the BZ in the points of which no ISWs (of the acoustic kind) occur in the spin-wave spectrum of the bilayer film. Whereas the shaded regions correspond to the set of points, where one ISW (less densely shaded regions) or respectively two ISWs (more densely shaded regions) exist. A perusal of Fig. 2 suffices to see that antiferromagnetic interface coupling considerably broadens the existence regions of ISWs; this is apparent, i.a. by the fact 
that compared to the ferromagnetic case the unshaded regions vanish completely. Secondly, the mapping topology exhibits symmetry: it is invariant with respect to a simultaneous change in sign of $D_{\text {int }}$ (that is, a change in type of the interface anisotropy) and a change in the magnetic field configuration (from perpendicular to parallel, or inversely). Thirdly, our mapping permits the general conclusion that ISW emergence is the easier the greater is the distance from the centre of the BZ and is the easiest at its boundaries and especially at its apices (corners), where predominantly both ISWs exist. The fourth conclusion to be drawn from Fig. 2 concerns the specific configuration with $\vartheta \approx 55^{\circ}$ (more exactly, $\vartheta=54.735^{\circ}$, for which $3 \cos ^{2} \vartheta=1$ ) - the only configuration at which the ISW mapping is completely insensitive to the type and magnitude of the interface anisotropy $D_{\text {int }}$.

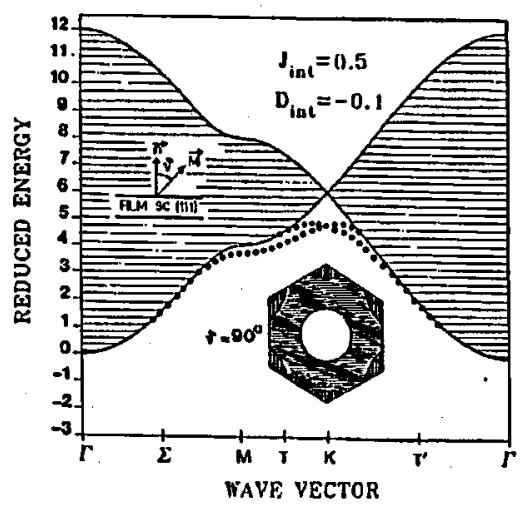

Fig. 3. The existence of the two ISW branches depicted in two ways: (i) by showing changes of their reduced energies (dotted lines) versus the in-plane vector $\boldsymbol{k}_{\|}$(varying along the high symmetry path of Fig. 1a), and (ii) by presenting the respective Brillouin zone mapping (inset) extracted from Fig. 2. Detailed explanation is given in the text. Continuous lines mark the upper and lower edges of the bulk band.

We shall now consider a selected case optionally chosen in order to give a practical illustration of the use of our map. We concentrate on the case when the BZ contains regions of all kinds: from Fig. 2 we select the sub-case corresponding to $J_{\text {int }}=+0.5, D_{\text {int }}=-0.1$ and $\vartheta=90^{\circ}$. For this case Fig. 3 shows the energy variations occurring in the ISWs spectrum as one proceeds along the path $\Gamma \rightarrow$ $\Sigma \rightarrow M \rightarrow T \rightarrow K \rightarrow T^{\prime} \rightarrow \Gamma$ (see Fig. 1) on the BZ; in fact, we are interested only in those details of the variations in energy that take place below the energy band, so in Fig. 3 we have marked (continuous lines) only the upper and lower edges of the band (without entering into details of the changes in energy of the bulk spin waves occurring within the band), whereas the dotted lines represent the changes in energy of the existing ISWs. We note that on the segment $\Gamma \rightarrow \Sigma$ no ISW branch exists (in the inset this corresponds to the unshaded BZ region); the first of them emerges in the vicinity of the point $\Sigma$ and remains the only one until we reach the point $T$, where a second ISW branch "is generated" (these branches correspond respectively to a symmetric and antisymmetric mode). Along 
the segment $T \rightarrow K$ the two branches approach each other and coalesce to a single degenerate energy level in the point $K$. To understand this effect let us note that in the point $K$ the structural factor $\left|\Gamma_{\perp}^{k_{11}}\right|$ (see Eq. (5b)) reaches zero value, and as a consequence of this the bulk band converges to a single level; the ISWs degeneracy arises in $K$ for the same reason (see Eq. (2)). Let us also note that the energy of the two ISWs, when degeneracy sets in, is the furthest downward shifted from the edge of the band, signifying that in the point $K$ the two modes achieve the strongest localization on the interface. The variations in ISW energy along the remaining path $K \rightarrow T^{\prime} \rightarrow \Gamma$ is easy to interpret in the inverse order: on returning to the $\Gamma$-point the ISWs are made to enter the band and finally the system is in the same conditions as at the outset.

Let us once again consider the existence condition for ISWs, Eq. (9). It involves both the sign of equality and that of inequality, the former corresponding to "generation" of ISW, i.e. to the situation when the respective mode leaves the band and becomes an ISW. At fixed values of $J_{\text {int }}, D_{\text {int }}$ and $\vartheta$ the condition for the sign of equality can be fulfilled by finding a set of vectors $\boldsymbol{k}_{\|}$(if extant) to which a set of points forming the boundary of the respective region of ISW existence corresponds on the BZ (e.g. a circle in Fig. 2). It is by now quite obvious that the topology of our BZ mapping is here the essential result. It portrays the boundaries of the regions of existence of the ISWs in their dependence on the parameters $J_{\text {int }}$, $D_{\text {int }}$ and the angle $\vartheta$, as well as the evolution of these boundaries under possible variations of the parameters.

\section{Case of $\operatorname{sc}(110)$ interface cut}

For the sc(110) interface cut the (acoustic) ISW reduced energy is given by the following formula:

$$
E_{\text {red }}^{\mathrm{ISW}}[\operatorname{sc}(110)]=6-2 \cos k_{1}-C_{ \pm}-\left(2 \cos \frac{1}{2} k_{2}\right)^{2} C_{ \pm}^{-1}
$$

where we have used the notation

$$
C_{ \pm}=2\left[1-J_{\text {int }}-D_{\text {int }}\left(3 \cos ^{2} \vartheta-1\right)\right] \pm 2 J_{\text {int }} \cos \frac{1}{2} k_{2}
$$

and the respective condition for the ISW existence reads

$$
\left[1-D_{\text {int }}\left(3 \cos ^{2} \vartheta-1\right)-J_{\text {int }}\right] \geq\left(1 \mp J_{\text {int }}\right) \cos \frac{1}{2} k_{2} \text {. }
$$

Figure 4 shows the Brillouin zone with, marked thereon, the existence regions of ISW determined from the condition (12) for three different orientations of the magnetization (with respect to the film normal) and six different combinations of (ferromagnetic/antiferromagnetic) interface coupling and (easy-axis/easy-plane) interface anisotropy. Figure 5 illustrates the changes in the reduced ISW energy spectrum versus the vector $k_{\|}$variation along the high symmetry BZ path for the case of antiferromagnetic interface coupling $\left(J_{\mathrm{int}}=-0.5\right)$. One notes that for the specific $v \approx 55^{\circ}$ configuration (for which the ISW energy and the existence condition are both insensitive to $D_{\text {int }}$ ), Fig. $5 \mathrm{~b}$, for $k_{\|}$from the centre of the zone $\left(\Gamma\right.$-point) there is one interface spin-wave, and with $k_{\|}$approaching some point between $X$ and $D$ the second ISW emerges. While the former ISW exists along 


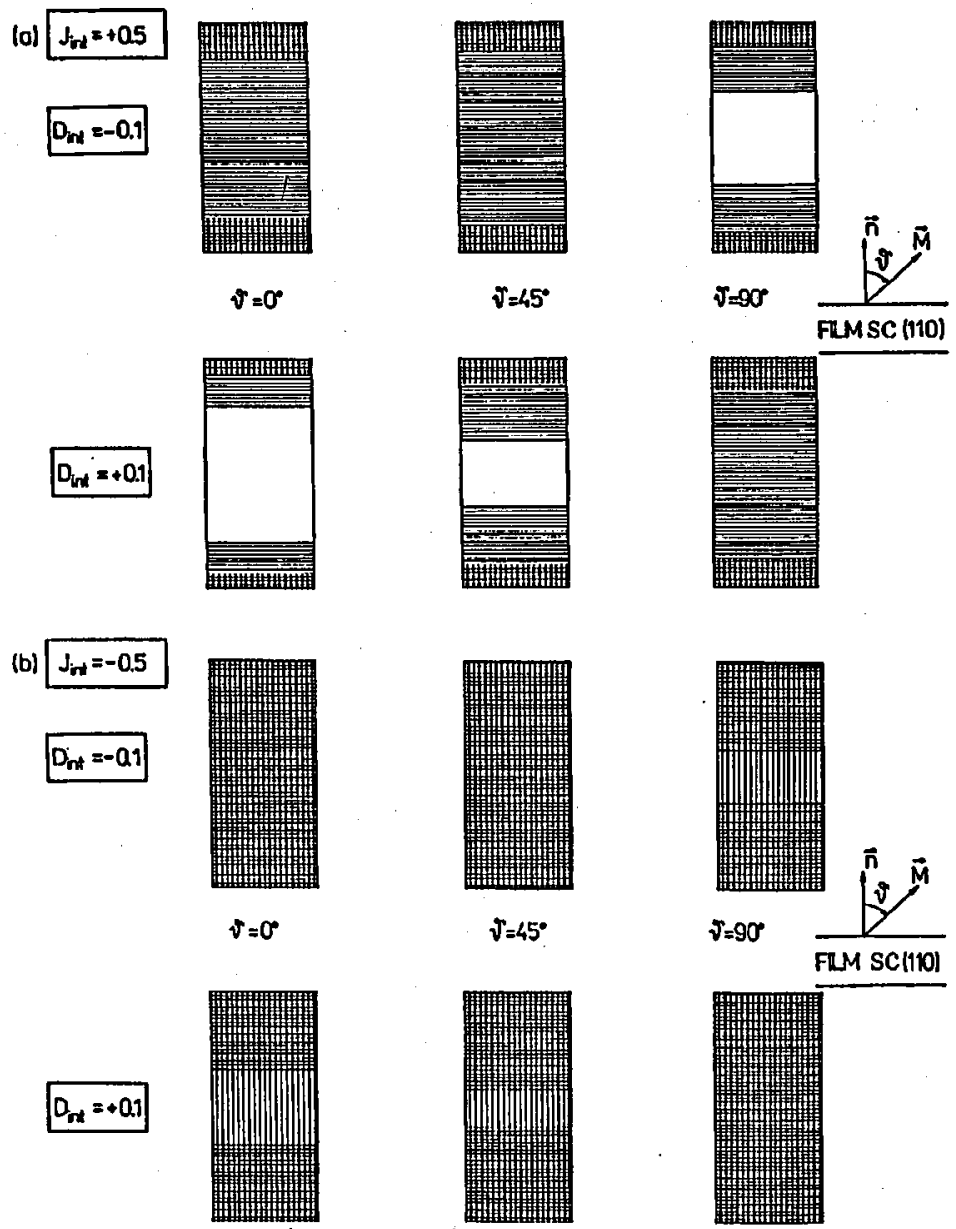

Fig. 4. Brillouin zone mapping of the regions of ISWs existence for bilayer sc(110) interface cut. The notations are the same as in Fig. 2.

the whole BZ path considered, the existence of the latter (i.e. the second) ISW is restricted only to a part of this path. However, one can cause the second ISW to exist also along the whole $\mathrm{BZ}$ path by changing the configuration and choosing an appropriate type (and value) of the interface anisotropy (see, Figs. 5a and 5c). Note also that in the region between $S$ and $Y$ two separated energy levels exist only: an ( $2 N-2)$-fold degenerate bulk level and, lying below it, a two-fold interface level. This interesting behaviour is a consequence of the vanishing of $\left|\Gamma_{\perp}^{k_{\| 1}}\right|$ for the propagation points between $S$ and $Y$. 

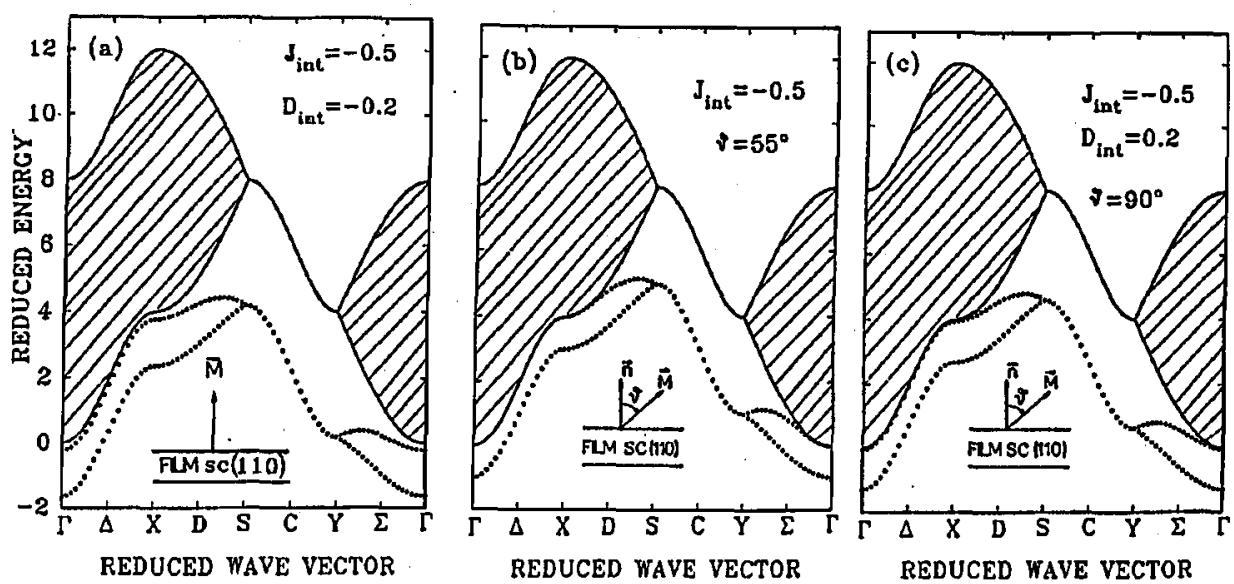

Fig. 5. Changes in the reduced ISWs energy (dotted lines) versus the in-plane vector $k_{\|}$varying along the high symmetry sc(110) BZ path (see, Fig. 1b) for the case of antiferromagnetic interface coupling and for three configurations of the film magnetization: (a) $\vartheta=0^{\circ}$, (b) $\vartheta \cong 55^{\circ}$ and (c) $\vartheta=90^{\circ}$. Explanation is given in the text.

\section{Interface spin-waves and rotation of the film magnetization. The critical angle effect}

Hitherto we have been studying the existence condition for ISWs, Eq. (6), concentrating on its dependence on the in-plane propagation vector $k_{\|}$. We shall now switch our attention to its dependence on $\vartheta$, the angle determining the configuration of the film magnetization (the other parameters including $\boldsymbol{k}_{\|}$being maintained constant). Here, we shall proceed quite generally in order to comprise all the interface cuts; the only graphical illustrations to be given will concern certain representative relations for the interface cut $\mathrm{sc}(110)$ which we select because its structural factors are of a particularly simple form facilitating our discussion. So let us now take a look at Eq. (6) assuming the angle $\vartheta$ as decisive for its fulfillment. The angle $\vartheta_{c}$ fulfilling the equality

$$
D_{\text {int }}\left(1-3 \cos ^{2} \vartheta\right)=\left(J_{\text {int }}-1\right)+\frac{1}{z_{\perp}}\left|\Gamma_{\perp}^{k_{\| 1}}\right|\left(1 \mp J_{\text {int }}\right)
$$

will be referred to as the critical angle. Any deviation from $\vartheta_{c}$ due to a rotation of the magnetization in the plane perpendicular to the film surface leads us into (or out of) the existence region of ISW (depending on the sense of the rotation). Thus, the critical angle $\vartheta_{\mathrm{c}}$ has the physical meaning of that particular configuration angle at which ISW emerges. Now let us note that in general Eq. (13) can give two critical angles, either of them corresponding to the emergence of the respective ISW. However, Eq. (13) can also altogether fail to give any critical angle. This can occur if either no ISWs exist in the whole range of variability of $\vartheta$, or inversely if ISWs exist for any value of $\vartheta$ (respectively, if the inequality (6) is - or is not fulfilled for each value of $\vartheta$ ).

The existence of a critical angle $\vartheta_{c}$ generally requires a specific set of values for the parameters $J_{\text {int }}, D_{\text {int }}$ and $k_{\|}$occurring in Eq. (13). We shall discuss this 
requirement for the case of sc(110) orientation, for which Eq. (13) takes the form

$$
1-3 \cos ^{2} \vartheta=\frac{J_{\mathrm{int}}-1}{D_{\mathrm{int}}} \times\left\{\begin{array}{l}
\left(1-\cos \frac{1}{2} k_{2}\right) \\
{\left[1+\left(\cos \frac{1}{2} k_{2}\right) \frac{J_{\mathrm{int}}+1}{J_{\mathrm{int}}-1}\right] .}
\end{array}\right.
$$

Figure 6 shows graphs of $\vartheta_{c}$ vs. $J_{\text {int }}$ determined with Eqs. (14) in the high symmetry point $X$ of the BZ (see Fig. 1b). On the basis of these graphs we select two pairs of values of $J_{\text {int }}$ and $D_{\text {int }}$ such as to provide for the existence of $t w o$ critical angles in the point $X$. For these cases, we show in Fig. 7 how the ISW energy varies with $\vartheta$ : at rotation of the magnetization (Fig. $7 \mathrm{a}-$ from normal to the plane; Fig. 7b - inversely) the two energetically lowest SW states lower their energy traversing the bottom of the band at their respective critical angles, subsequently entering the "forbidden" region and thus becoming ISW states. (We draw the Reader's attention to the fact that the definition of our quantity called the ISW reduced energy ensures that the bulk band edges are constant with regard to $\vartheta$, as in fact it is seen in Fig. 7.)
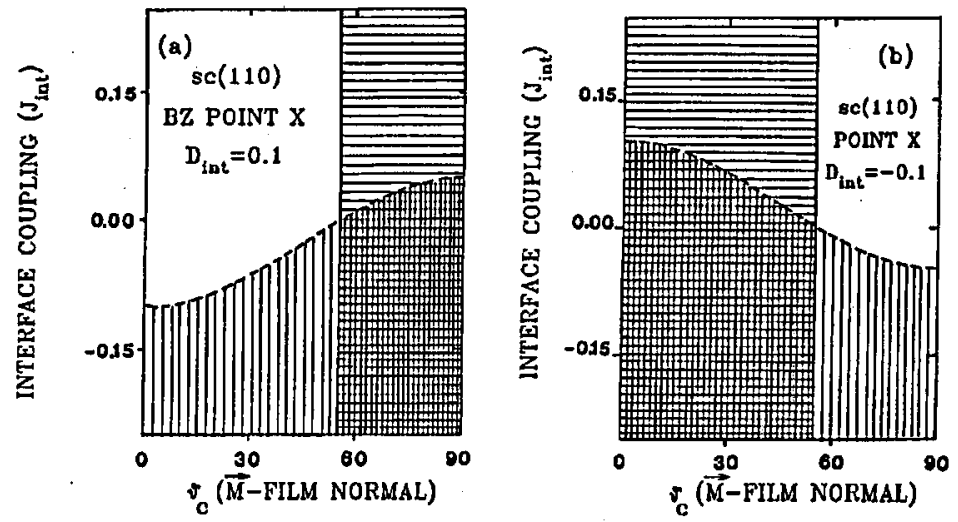

Fig. 6. Illustration of the critical angle $\left(\vartheta_{c}\right)$ dependence on the interface coupling $\left(J_{\text {int }}\right)$, as defined by Eqs. (14), depicted for sc(110) interface cut in the BZ point $X$ and for (a) easy-axis interface anisotropy and (b) for that of the easy-plane type. The vertical solid line corresponds to Eq. (14a) (which gives the only value $\vartheta \approx 55^{\circ}$ ); the dotted line corresponds to Eq. (14b). Two interface waves exist in the more densely shaded region, one - in the less densely shaded regions, and none - in the unshaded area.

How, on physical grounds, are we to understand the phenomenon of a critical angle? The existence of a critical angle is rendered possible by the presence of uniaxial interface anisotropy in our model, contributing to the pinning energy of the interface spins. If this contribution frees the interface spins from their pinning in the direction of the static magnetization (of the film as a whole), ISWs are called into existence, and $\vartheta_{\mathbf{c}}$ is precisely that specific angle for which the tendency of the interface spins to "freedom" begins to outweigh their tendency to "pinning". However, it is essential to note that if antiferromagnetic interface coupling is very 

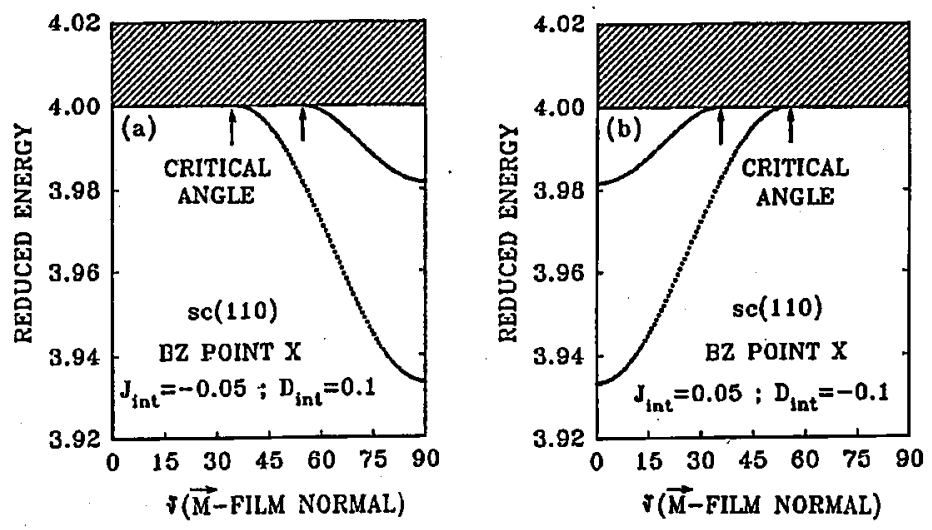

Fig. 7. The dependence of the ISWs reduced energies (dotted lines: decreasing (a) or increasing (b) type) on the configuration angle $\vartheta$ of the film magnetization (the shaded area indicates the bottom of the bulk band). Note the existence of two critical angles (indicated by arrows) of ISWs emergence.

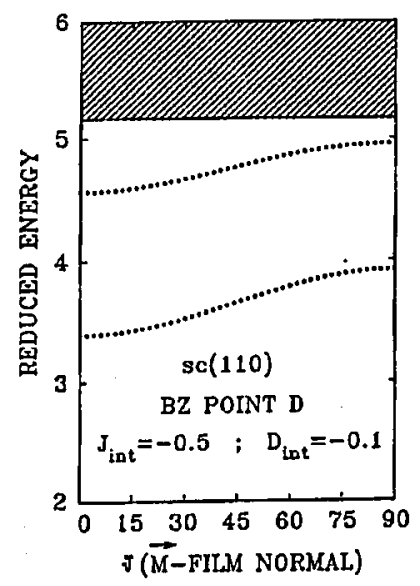

Fig. 8. The two interface spin-wave energy branches plotted against the configuration angle $\vartheta$ of the film magnetization for the case when no critical angles exist. The shaded area indicates the bottom of the bulk band.

strong, the interface spins are already sufficiently free to ensure the existence of both ISWs in the whole range of variability of $\vartheta$ and no critical angle effect takes place (see Fig. 8).

The novel properties of the function $E_{\text {red }}^{\text {ISW }}(\vartheta)$ derived in the present section seem interesting in that they open the way for experimental exploration. We shall draw attention to these outlooks in the next section. 


\section{Final remarks and experimental implications}

Let us consider the experimental conditions favouring the occurrence of a critical angle $\vartheta_{\mathrm{c}}$. The magnitude of $\vartheta_{\mathrm{c}}$ is determined by Eq. (13) which generally gives two critical angles, respectively for the sign "+" or "-" as the case may be:

$$
\begin{aligned}
& D_{\text {int }}\left(1-3 \cos ^{2} \vartheta_{\mathrm{c} 1}\right)=\left(J_{\mathrm{int}}-1\right)\left(1-\frac{1}{z_{\perp}}\left|\Gamma_{\perp}^{k_{\|}}\right|\right), \\
& D_{\mathrm{int}}\left(1-3 \cos ^{2} \vartheta_{\mathrm{c} 2}\right)=\left(J_{\mathrm{int}}-1\right)\left(1+\frac{1}{z_{\perp}}\left|\Gamma_{\perp}^{k_{\|}}\right| \frac{J_{\mathrm{int}}+1}{J_{\mathrm{int}}-1}\right) .
\end{aligned}
$$

Clearly, the concept of a critical angle is meaningful only if $D_{\text {int }} \neq 0$. For fixed values of $J_{\text {int }}$ and $D_{\text {int }}$, the angle $\vartheta_{c}$ (if present) is a function of the in-plane propagation vector $k_{\|\|}$. We start by considering the situation in the centre of the $\mathrm{BZ}$, that is, for $k_{\|}=0$; Eqs. (15) become now as follows:

$$
D_{\text {int }}\left(1-3 \cos ^{2} \vartheta_{c}\right)=\left\{\begin{array}{l}
0 \\
2 J_{\text {int }} .
\end{array}\right.
$$

The first of them gives a critical angle equal to $\vartheta_{\mathrm{c} 1}=54.735^{\circ}$ irrespective of the values of $D_{\text {int }}$ and $J_{\text {int }}$. The other gives a second critical angle albeit only if $J_{\text {int }}$ and $D_{\text {int }}$ are comparable as to their absolute values. However, since the interface anisotropy parameter $D_{\text {int }}$ is, at the most, of the order of $10^{-3}$, the second critical angle $\left(\vartheta_{\mathrm{c} 2}\right)$ can be searched for only in the narrow range of variability of the interlayer spacer thickness, where $J_{\text {int }}$ exhibits a change in sign; the oscillations of $J_{\text {int }}$ with varying thickness of the interlayer spacer have been studied rather extensively for a variety of materials (see, e.g. [6]), so that the practical determination of the range of spacer thickness where $\left|J_{\text {int }}\right| \approx\left|D_{\text {int }}\right|$ should involve no serious problems for the experimenter. This, in fact, is the range of thickness of the spacer, where the second critical angle $\vartheta_{\mathrm{c} 2}$ should be searched for. If both critical angles exist, the one always corresponds to the creation of a symmetric ISW and the other to that of an antisymmetric ISW (whereas a single critical angle may correspond to the creation of the one or the other kind of ISW).

A similar analysis of Eqs. (15) can be carried out for any arbitrarily selected point of the $\mathrm{BZ}$ with a propagation vector $k_{\|} \neq 0$. If one of the Eqs. (15) has a solution $\vartheta_{\mathrm{c}}$ in the $\mathrm{BZ}$ point selected, there will exist a specific orientation of the magnetization of the sample ("configuration $\vartheta_{\mathrm{c}}$ ") which, if traversed (by performing a rotation of $M$ ), will restrict the in-plane propagation of the spin-wave to the interface region of the sample, the region in question being the narrower the farther remote is $M$ from the critical configuration $\vartheta_{\mathrm{c}}$. Now one is led to expect that this effect of statically induced magnon propagation on the interface can prove useful in certain applications to spin-wave electronics (see e.g. [7]).

Let us now consider how the effects due to the presence of critical angles can be exploited in measurements of quantities characterizing the interface. By Eqs. (15), the angles under consideration are functions of $k_{\|}, J_{\text {int }}$ and $D_{\text {int }}$ :

$$
\begin{aligned}
& \vartheta_{\mathrm{c} 1}=\vartheta_{\mathrm{c} 1}\left(k_{\|}, J_{\mathrm{int}}, D_{\mathrm{int}}\right), \\
& \vartheta_{\mathrm{c} 2}=\vartheta_{\mathrm{c} 2}\left(k_{\|}, J_{\mathrm{int}}, D_{\mathrm{int}}\right) .
\end{aligned}
$$


Since two experimentally measurable quantities and two equations defining them are available, we are potentially in a position to determine the two interface quantities $J_{\text {int }}$ and $D_{\text {int }}$. However, we have to take into account that depending on the measuring method at our disposal it may prove impossible to determine the two characteristic angles $\vartheta_{\mathrm{c} 1}$ and $\vartheta_{\mathrm{c} 2}$ in a single experiment. Nonetheless we should draw attention to the feasibility of applying Eqs. (17) in different experimental setups permitting the determination of $J_{\text {int }}$ and $D_{\text {int }}$ separately.

Concerning the choice of an experimental procedure enabling the measurement of those effects, we have clarity only regarding the situation restricted to ISW in the centre of the BZ, i.e. for $k_{\|}=0$ (in fact, we then deal with interface standing modes (ISM)). The latter can be studied applying the ferromagnetic resonance (FMR) method [8] enabling the taking of resonance spectra at different configurations of the static field and thus the study of the angular effects under consideration (effects due to the critical angle in bilayer FMR have been discussed extensively earlier $[2,9,10])$. For the experimental detection of ISW in points of the $\mathrm{BZ}$ where $k_{\|} \neq 0$ one should expect Brillouin light scattering to be applicable [11-13], especially two-magnon scattering - since it allows us to penetrate wave vectors distributed throughout the entire BZ [14]. Another technique, which hopefully can be used to probe ISWs, is inelastic neutron scattering. However, we have no certainty as to whether measurable detection can be achieved by those two methods on such a small volume of the material as the "interface region".

Finally, let us note that an effect of critical angle exists as well in the excitation of surface spin-waves $[8,15]$ and that its physical nature resembles that of the interface effects considered in the present work. Hence we expect the concept of the interface critical angle discussed here may prove equally fruitful as that of the surface critical angle [16].

\section{Acknowledgments}

One of the authors (IIP) wishes to express his gratitude to the Laboratoire de Dynamique et Structure des Matériaux Moléculaires, Unité Associée au CNRS 801, Université de Lille I, for their hospitality. Thanks are also due tothe Committee for Scientific Research for its support under the grant No. 2 P03B 04308.

\section{References}

[1] H. Puszkarski, A. Akjouj, B. Djafari-Rouhani, L. Dobrzynski, Phys. Rev. B 51, 16008 (1995).

[2] H. Puszkarski, Surf. Sci. Rep. 20, 45 (1994).

[3] B. Kołodziejczak, M. Sc. Thesis, Poznań University, Poznan 1991.

[4] B. Kołodziejczak, H. Puszkarski, Acta Phys. Pol. A 80, 675 (1991).

[5] B. Kołodziejczak, H. Puszkarski, Acta Phys. Pol. A 83, 661 (1993).

[6] Magnetism and Structure in Systems of Reduced Dimension, Eds. R.F.C. Farrow, B. Dieny, M. Donath, A. Fert, B.D. Hermsmeier, NATO ASI Series B Physics, Vol. 309, Plenum Press, New York 1993.

[7] G. Prinz, K. Hathaway, Phys. Today (Special Issue: Magnetoelectronics) 48, 24 (1995). 
[8] H. Puszkarski, Prog. Surf. Sci. 9, 191 (1979).

[9] H. Puszkarski, J. Phys., Condens. Matter 6, 1155 (1994).

[10] H. Puszkarski, to be published.

[11] P. Grünberg, in: Light Scattering in Solids V, Eds. M. Cardona, G. Güntherodt, in Springer series Topics in Applied Physics, Springer, Berlin 1989.

[12] P. Kabos, C.E. Patton, M.O. Dima, D.B. Church, R.L. Stamps, R.E. Camley, J. Appl. Phys. 75, 3553 (1994).

[13] B. Hillebrands, in: Light Scattering in Solids VII, Eds. M. Cardona, G. Güntherodt, in Springer series Topics in Applied Physics, Springer, Berlin 1993.

[14] M.G. Cottam, D.J. Lockwood, Light Scattering in Magnetic Solids, J. Wiley and Sons, New York 1986.

[15] H. Puszkarski, Acta Phys. Pol. A 38, 217, 899 (1970).

[16] J.T. Yu, R.A. Turk, P.E. Wigen, Phys. Rev. B 11, 420 (1975). 